\title{
Some unexplored features of the nonlinear compressive magnetoacoustic Alfvénic waves
}

\author{
J. Vranjes ${ }^{1}$, B. P. Pandey ${ }^{2}$ \\ ${ }^{1}$ Institute of Physics Belgrade, Pregrevica 118, 11080 Zemun, Serbia. \\ ${ }^{2}$ Department of Physics \& Astronomy \& Research Centre for Astronomy, \\ Astrophysics \& Astrophotonics, Macquarie University, Sydney, NSW 2109, Australia. \\ E-mail: jvranjes@yahoo.com; drbp.pandey@gmail.com
}

\begin{abstract}
The theory of nonlinear magnetoacoustic wave in the past has strictly been focused on purely compressive features of the mode. We show that a complete set of nonlinear equations necessarily includes both compressional and shear components of the magnetic field. These two turn out to be described by exactly the same nonlinear equations, which make the use of such a complete full set of equations far less complicated than expected. Present results should considerably enrich the theory of these waves by opening up new frontiers of investigation and providing some completely new types of nonlinear solutions.
\end{abstract}

PACS numbers: 52.30.Ex, 52.35.Mw, 52.35.Sb, 52.35.Tc, 52.35.Bj 


\section{Introduction}

There have been many studies of the nonlinear hydro-magnetic waves in the past half a century of plasma science. This is not surprising in view of the fact that such waves are expected in various environments in the laboratory, space and astrophysical plasmas. The theory of such waves can be traced as far back as to 1958 [1], and it can be seen in many studies ever since [2]-[27].

In the papers [1]-[27] electromagnetic perturbations propagating perpendicular to the magnetic field are assumed to be purely compressible (i.e., having the perturbed magnetic field component only along the ambient magnetic field vector). Within the linear theory, such purely compressive magnetic perturbations indeed follow from the geometry of the mode, and this holds without any assumption. However, within the nonlinear theory an additional perpendicular shear component of the magnetic field appears naturally. This perpendicular component turns out to be described by an equation which is exactly the same as the equation for the compressional component, see later in the text. Nevertheless, the usual nonlinear theory [1]-[27] dealing with purely compressive phenomena is formally correct, such compressive nonlinear solutions are mathematically allowed and physically possible. Physical phenomena obtained within such a model are numerous and this is partly seen from the huge number of studies in the past fifty years, and after so many decades the mode is still in the focus of researchers as it was in its early days, c.f. recent Refs. [18]-[27]. The unexplored physics of the nonlinear mode, that should follow from the additional shear component, is expected to be important.

In this work a closed set of nonlinear equations for the generalized magnetoacoustic mode will be derived showing that both compressible and shear components of the magnetic field are described by an exactly the same nonlinear equation. One simple solution of such a complete set of equations will be presented in order to show that such a generalized theory can be used in a manner very similar to the usual studies where only the compressional part of the magnetic field is taken into account.

\section{Model and equations}

The model which we describe here uses an arbitrary background magnetic field in the $z$-direction $\vec{B}_{0}=B_{0} \vec{e}_{z}$. Further, we shall use two-fluid description for the electrons and ions that include, respectively, the momentum equations for the two species $j=e, i$, and the Faraday and Ampère laws:

$$
\begin{aligned}
& \frac{D_{e} \vec{v}_{e}}{D t}=-\frac{e}{m_{e}} \vec{E}-\nabla p_{e}-\frac{e}{m_{e}} \vec{v}_{e} \times \vec{B}-\nu_{e i}\left(\vec{v}_{e}-\vec{v}_{i}\right), \\
& \frac{D_{i} \vec{v}_{i}}{D t}=\frac{e}{m_{i}} \vec{E}-\nabla p_{i}+\frac{e}{m_{i}} \vec{v}_{i} \times \vec{B}-\nu_{i e}\left(\vec{v}_{i}-\vec{v}_{e}\right), \\
& \nabla \times \vec{E}=-\frac{\partial \vec{B}}{\partial t}
\end{aligned}
$$


Nonlinear compressive magnetoacoustic Alfvénic waves

$$
\nabla \times \vec{B}=\mu_{0} \vec{j}=\mu_{0} e\left(n_{i} \vec{v}_{i}-n_{e} \vec{v}_{e}\right) .
$$

In Eqs. (1)-(4) singly charged ions (i.e., protons) are assumed. We shall use the quasineutrality condition:

$$
n_{e}=n_{i}=n,
$$

and the continuity equation for ions

$$
\frac{\partial n}{\partial t}+\nabla \cdot\left(n \vec{v}_{i}\right)=0 .
$$

We shall discuss perturbations propagating through a homogeneous plasma in the $x$ direction, perpendicular to the ambient magnetic field so that $D_{j} / D t \equiv \partial / \partial t+v_{j x} \partial / \partial x$.

To point out some basic properties of the mode, in this part we omit collisions, and assume inertia-less electrons and isothermal and quasi-neutral perturbations $p_{j 1}=$ $\kappa T_{j} n_{1}$. Within these assumptions, from linearized Eq. (1), one may calculate the electric field $\vec{E}_{1}$

$$
\vec{E}_{1}=-\frac{\kappa T_{e}}{e n_{0}} \nabla n_{1}-\vec{v}_{e 1} \times \vec{B}_{0},
$$

and plug it in the momentum equation for ions, which, with the help of linearized Ampère law [Eq. 目 consequently becomes

$$
m_{i} n_{0} \frac{\partial \vec{v}_{i 1}}{\partial t}=\frac{1}{\mu_{0}}\left(\nabla \times \vec{B}_{1}\right) \times \vec{B}_{0}-\kappa\left(T_{i}+T_{e}\right) \nabla n_{1} .
$$

Taking the curl of Eq. (7) and expressing $\vec{v}_{e 1}$ from the linearized Eq. (44) with the help of Eq. (3) one obtains the following induction equation

$$
\frac{\partial \vec{B}_{1}}{\partial t}=\nabla \times\left(\vec{v}_{i 1} \times \vec{B}_{0}\right)-\nabla \times\left[\frac{1}{\mu_{0} e n_{0}}\left(\nabla \times \vec{B}_{1}\right) \times \vec{B}_{0}\right] .
$$

The linear set of equations is closed with the linearized version of Eq. (6) . For perturbations propagating in the $x$-direction $\nabla \equiv \vec{e}_{x} \partial / \partial x$, the first term on the right-hand side in (9) yields $-B_{0} \vec{e}_{z} \partial v_{i x 1} / \partial x$, while the second term vanishes. Hence, Eq. (9) yields only the $z$-component of $\vec{B}_{1}$. Similarly, the first term on the right-hand side in Eq. (8) also contains only the $z$-component of $\vec{B}_{1}$ because $\left(\nabla \times \vec{B}_{1}\right) \times \vec{B}_{0}=$ $-\vec{e}_{x} B_{0} \partial B_{z 1} / \partial x$.

Within the linear regime, equations consequently yield the hydrodynamically longitudinal $\left(\vec{v}_{i 1} \equiv v_{i x 1} \vec{e}_{x}\right)$ and electro-dynamically transverse $\left(\vec{B}_{1} \equiv B_{z 1} \vec{e}_{z}, \vec{E}_{1} \equiv \vec{E}_{y 1} \vec{e}_{y}\right)$ purely compressive perturbations of the magnetic field, which describe the fast magnetoacoustic mode with the frequency $\omega^{2}=k_{x}^{2}\left(c_{s}^{2}+c_{a}^{2}\right)$, where $c_{s}^{2}=\kappa\left(T_{e}+T_{i}\right) / m_{i}$, and $c_{a}^{2}=B_{0}^{2} /\left(\mu_{0} m_{i} n_{0}\right)$. This purely compressive nature of the mode within the linear theory is therefore not assumed, but it simply follows from the geometry of the mode.

However, within the nonlinear theory, the mode does not necessarily remain purely compressive as will be demonstrated below. This important feature has not been explored in the past. It will be shown below that the generalization of the theory is in fact rather straightforward, and it only includes one additional nonlinear equation 
for the perpendicular magnetic field which is, within the given approximations, identical to the equation for its compressive component.

In the nonlinear regime, the term $(\nabla \times \vec{B}) \times \vec{B}$ in the corresponding nonlinear counterpart of Eq. (8) reads

$$
-\vec{e}_{x}\left(B_{z} \frac{\partial B_{z}}{\partial x}+B_{y} \frac{\partial B_{y}}{\partial x}\right)+\vec{e}_{y} B_{x} \frac{\partial B_{y}}{\partial x}+\vec{e}_{z} B_{x} \frac{\partial B_{z}}{\partial x} .
$$

While writing this expression, we have dropped the subscript 1 from the perturbed quantities. Clearly, there is an additional perpendicular component $B_{y}$ which cannot be omitted now. Hence, simply assuming purely compressive perturbations in the nonlinear regime may not always be justified. We shall repeat the derivations by keeping all the terms in the Eqs. (1)-(6), i.e., by retaining the finite electron inertia and collision terms.

Eq. (7) in the presence of inertia and collision terms now reads

$$
\vec{E}=-\frac{\kappa T_{e}}{e n} \nabla n-\vec{v}_{e} \times \vec{B}-\frac{\nu_{e i} m_{e}}{e}\left(\vec{v}_{e}-\vec{v}_{i}\right)-\frac{m_{e}}{e} \frac{D_{e} \vec{v}_{e}}{D t} .
$$

This is used in Eq. (2) together with the Ampère law Eq. (4), and, with the momentum conservation which in the quasi-neutral system like the present one implies $\nu_{i e}=$ $m_{e} \nu_{e i} / m_{i}$. The ion momentum equation now becomes

$$
\frac{\partial \vec{v}_{i}}{\partial t}+\left(\vec{v}_{i} \cdot \nabla\right) \vec{v}_{i}=\frac{1}{\mu_{0} m_{i} n}(\nabla \times \vec{B}) \times \vec{B}-c_{s}^{2} \frac{\nabla n}{n}-\frac{m_{e}}{m_{i}} \frac{D_{e} \vec{v}_{e}}{D t} .
$$

The electron velocity in Eq. (11) is eliminated by using Eq. (41)

$$
\begin{aligned}
& \frac{D_{e} \vec{v}_{e}}{D t}=\left[\frac{\partial}{\partial t}+\left(\vec{v}_{i}-\frac{\nabla \times \vec{B}}{\mu_{0} e n}\right) \cdot \nabla\right]\left(\vec{v}_{i}-\frac{\nabla \times \vec{B}}{\mu_{0} e n}\right) \\
& \equiv\left(\frac{\partial}{\partial t}+\vec{v}_{i} \cdot \nabla\right)\left(\vec{v}_{i}-\frac{\nabla \times \vec{B}}{\mu_{0} e n}\right)=\frac{m_{e}}{m_{i}} \frac{D_{i} \vec{v}_{i}}{D t}-\frac{m_{e}}{m_{i}} \frac{D_{i}}{D t}\left(\frac{\nabla \times \vec{B}}{\mu_{0} e n}\right),
\end{aligned}
$$

where we have used the fact that $(\nabla \times \vec{B}) \cdot \nabla=\left[-\vec{e}_{y} \partial B_{z} /(\partial x)+\vec{e}_{z} \partial B_{y} /(\partial x)\right] \cdot \nabla \equiv 0$. Eq. (11) becomes

$$
\frac{\partial \vec{v}_{i}}{\partial t}+\left(\vec{v}_{i} \cdot \nabla\right) \vec{v}_{i}=\frac{1}{\mu_{0} m_{i} n}(\nabla \times \vec{B}) \times \vec{B}-c_{s}^{2} \frac{\nabla n}{n}+\frac{m_{e}}{m_{i}} \frac{D_{i}}{D t}\left(\frac{\nabla \times \vec{B}}{\mu_{0} e n}\right) .
$$

The only approximation used so far is

$$
1+m_{e} / m_{i} \simeq 1
$$

Applying $\nabla \times$ onto Eq. (10) and using Eqs. (3, 4) yields the following induction equation

$$
\begin{aligned}
& \frac{\partial \vec{B}}{\partial t}=\nabla \times\left[\left(\vec{v}_{i} \times \vec{B}\right)-\frac{1}{\mu_{0} e}\left(\frac{\nabla \times \vec{B}}{n} \times \vec{B}\right)\right] \\
& -\frac{\nu_{e i} m_{e}}{\mu_{0} e^{2}} \nabla \times\left(\frac{\nabla \times \vec{B}}{n}\right)+\frac{m_{e}}{e} \nabla \times \frac{D_{e} \vec{v}_{e}}{D t} .
\end{aligned}
$$


In the above induction equation, along with the advection, and Hall and Ohm terms, the correction due to electron inertia has been retained on the right-hand side.

We use Eq. (12) to calculate the last term in Eq. (15), and this yields

$$
\begin{aligned}
& \frac{\partial \vec{B}}{\partial t}=-\vec{e}_{y} \frac{\partial}{\partial x}\left(v_{i x} B_{y}-v_{i y} B_{x}\right)+\vec{e}_{z} \frac{\partial}{\partial x}\left(v_{i z} B_{x}-v_{i x} B_{z}\right) \\
& +\frac{\vec{e}_{y}}{\mu_{0} e} \frac{\partial}{\partial x}\left(\frac{B_{x}}{n} \frac{\partial B_{z}}{\partial x}\right)-\frac{\vec{e}_{z}}{\mu_{0} e} \frac{\partial}{\partial x}\left(\frac{B_{x}}{n} \frac{\partial B_{y}}{\partial x}\right) \\
& +\frac{m_{e} \nu_{e i}}{\mu_{0} e^{2}}\left[\vec{e}_{y} \frac{\partial}{\partial x}\left(\frac{1}{n} \frac{\partial B_{y}}{\partial x}\right)+\vec{e}_{z} \frac{\partial}{\partial x}\left(\frac{1}{n} \frac{\partial B_{z}}{\partial x}\right)\right] \\
& +\frac{m_{e}}{e}\left\{\frac{\partial}{\partial t}\left(-\vec{e}_{y} \frac{\partial v_{i z}}{\partial x}+\vec{e}_{z} \frac{\partial v_{i y}}{\partial x}\right)-\vec{e}_{y} \frac{\partial}{\partial x}\left(v_{i x} \frac{\partial v_{i z}}{\partial x}\right)+\vec{e}_{z} \frac{\partial}{\partial x}\left(v_{i x} \frac{\partial v_{i y}}{\partial x}\right)\right. \\
& +\frac{1}{\mu_{0} e} \frac{\partial}{\partial t}\left[\vec{e}_{y} \frac{\partial}{\partial x}\left(\frac{1}{n} \frac{\partial B_{y}}{\partial x}\right)+\vec{e}_{z} \frac{\partial}{\partial x}\left(\frac{1}{n} \frac{\partial B_{z}}{\partial x}\right)\right] \\
& \left.+\frac{1}{\mu_{0} e} \vec{e}_{y} \frac{\partial}{\partial x}\left[v_{i x} \frac{\partial}{\partial x}\left(\frac{1}{n} \frac{\partial B_{y}}{\partial x}\right)\right]+\frac{1}{\mu_{0} e} \vec{e}_{z} \frac{\partial}{\partial x}\left[v_{i x} \frac{\partial}{\partial x}\left(\frac{1}{n} \frac{\partial B_{z}}{\partial x}\right)\right]\right\} .
\end{aligned}
$$

From this equation we conclude that

$$
B_{x}=0,
$$

and thus, the Hall term drops out of the equation. The two remaining components of the magnetic field are consequently described by

$$
\begin{aligned}
& \frac{\partial B_{y}}{\partial t}+\frac{\partial}{\partial x}\left(v_{i x} B_{y}\right)=-\frac{m_{e}}{e} \frac{\partial}{\partial x}\left(\frac{\partial}{\partial t}+v_{i x} \frac{\partial}{\partial x}\right) v_{i z}+\frac{m_{e} \nu_{e i}}{\mu_{0} e^{2}} \frac{\partial}{\partial x}\left(\frac{1}{n} \frac{\partial B_{y}}{\partial x}\right) \\
& +\frac{m_{e}}{\mu_{0} e^{2}} \frac{\partial}{\partial x}\left(\frac{\partial}{\partial t}+v_{i x} \frac{\partial}{\partial x}\right)\left(\frac{1}{n} \frac{\partial B_{y}}{\partial x}\right), \\
& \frac{\partial B_{z}}{\partial t}+\frac{\partial}{\partial x}\left(v_{i x} B_{z}\right)=\frac{m_{e}}{e} \frac{\partial}{\partial x}\left(\frac{\partial}{\partial t}+v_{i x} \frac{\partial}{\partial x}\right) v_{i y}+\frac{m_{e} \nu_{e i}}{\mu_{0} e^{2}} \frac{\partial}{\partial x}\left(\frac{1}{n} \frac{\partial B_{z}}{\partial x}\right) \\
& +\frac{m_{e}}{\mu_{0} e^{2}} \frac{\partial}{\partial x}\left(\frac{\partial}{\partial t}+v_{i x} \frac{\partial}{\partial x}\right)\left(\frac{1}{n} \frac{\partial B_{z}}{\partial x}\right) .
\end{aligned}
$$

Within the approximation (14), the first terms on the right-hand sides in Eqs. (18, 19) can be neglected. This may be seen from the following comparison of terms in (18). We compare the left-hand side [the term $a$ ] with the first term on the RHS [the term $b$ ], and the result is

$$
\frac{a}{b}=e B_{y} /\left(m_{e} k v_{i z}\right)
$$

Here and further we use Eq. (23) (see further in the text) which yields

$$
v_{i z} \simeq \frac{m_{e}}{m_{i}} \frac{k B_{y}}{\mu_{0} e n}
$$


Hence, we have

$$
\frac{a}{b}=\frac{m_{i}}{m_{e}} \frac{1}{k^{2} \lambda_{e}^{2}} \gg 1, \quad \lambda_{e}=c / \omega_{p e} .
$$

Now compare the term $b$ with the last term in (18):

$$
\frac{b}{c} \simeq \frac{\mu_{0} e n v_{i z}}{k B_{y}}=\frac{m_{e}}{m_{i}} .
$$

Finally, compare $b$ with the second term on the rhs in (18):

$$
\frac{b}{d}=\frac{\mu_{0} e n \omega v_{i z}}{\nu_{e i} k B_{y}}=\frac{m_{e}}{m_{i}} \frac{\omega}{\nu_{e i}} .
$$

Here $\omega / \nu_{e i}$ is in principle arbitrary but strictly speaking it should be below 1 in order to justify the use of fluid theory. In view of the mass difference we may conclude that the first term on the rhs in (18) is indeed negligible. A similar comparison of terms can be used in Eq. (19), showing that the first term on the rhs in Eq. (19) is negligible as well.

Consequently, what remains are two identical equations for both the shear and compressional components of the magnetic field $B_{y}, B_{z}$, obtained within the same approximations:

$$
\begin{aligned}
& \frac{\partial B_{\alpha}}{\partial t}+\frac{\partial}{\partial x}\left(v_{i x} B_{\alpha}\right)=\frac{m_{e} \nu_{e i}}{\mu_{0} e^{2}} \frac{\partial}{\partial x}\left(\frac{1}{n} \frac{\partial B_{\alpha}}{\partial x}\right) \\
& +\frac{m_{e}}{\mu_{0} e^{2}} \frac{\partial}{\partial x}\left(\frac{\partial}{\partial t}+v_{i x} \frac{\partial}{\partial x}\right)\left(\frac{1}{n} \frac{\partial B_{\alpha}}{\partial x}\right), \quad \alpha=y, z .
\end{aligned}
$$

In view of Eq. (17), from Eq. (13) we have the following equations for the ion velocity components

$$
\begin{aligned}
& \left(\frac{\partial}{\partial t}+v_{i x} \frac{\partial}{\partial x}\right) v_{i x}=-c_{s}^{2} \frac{1}{n} \frac{\partial n}{\partial x}-\frac{1}{2 \mu_{0} m_{i} n} \frac{\partial}{\partial x}\left(B_{y}^{2}+B_{z}^{2}\right) \\
& \left(\frac{\partial}{\partial t}+v_{i x} \frac{\partial}{\partial x}\right) v_{i y}=-\frac{1}{\mu_{0} e} \frac{m_{e}}{m_{i}}\left(\frac{\partial}{\partial t}+v_{i x} \frac{\partial}{\partial x}\right)\left(\frac{1}{n} \frac{\partial B_{z}}{\partial x}\right) \\
& \left(\frac{\partial}{\partial t}+v_{i x} \frac{\partial}{\partial x}\right) v_{i z}=\frac{1}{\mu_{0} e} \frac{m_{e}}{m_{i}}\left(\frac{\partial}{\partial t}+v_{i x} \frac{\partial}{\partial x}\right)\left(\frac{1}{n} \frac{\partial B_{y}}{\partial x}\right)
\end{aligned}
$$

The ion continuity rewritten here reads

$$
\frac{\partial n}{\partial t}+\frac{\partial}{\partial x}\left(n v_{i x}\right)=0
$$

Within the approximation (14), the equations (21), (24) together with the two equations (20) make a complete closed set. This set of equations contains both the compressional and the perpendicular components of the magnetic field $B_{z}, B_{y}$.

Observe that Eq. (20) is formally correctly satisfied with the trivial case $B_{y} \equiv 0$, which then reduces the equations to those studied so far in Refs. [1]-[27]. However, the magnetic field equation is identical for both $B_{y}$ and $B_{z}$, and thus the same can be 
done with the $B_{z} \equiv 0$, and what remains is a closed set which determines the nonlinear perpendicular (shear) component of the magnetic field $B_{y}$, with a plethora of possible solutions that have not been explored in the past. This is even more so if the nontrivial case is studied for both components. Clearly, neglecting one or the other component without justification may lead to unphysical singular solution.

In some cases, solving the complete set of equations (20), (21), (24) can be rather straightforward, and it can easily be reduced to the procedure used for the purely compressive perturbations. This is most clearly seen in the case of cold plasma and in the limit of massless electrons. From Eqs. (20), (24) we obtain the frozen-in condition for both components $B_{y}, B_{z}$ separately:

$$
\begin{aligned}
& B_{z}=a_{1} n, \\
& B_{y}=a_{2} n,
\end{aligned}
$$

where $a_{1}, a_{2}$ are arbitrary constants. This further implies that $B_{y}=a_{3} B_{z}$; using this in Eq. (21) we have

$$
\frac{\partial v_{i x}}{\partial t}+v_{i x} \frac{\partial v_{i x}}{\partial x}+c_{1} \frac{\partial B_{z}}{\partial x}=0, \quad c_{1}=\frac{a_{1}\left(a_{3}^{2}+1\right)}{\mu_{0} m_{i}} .
$$

This equation is coupled with

$$
\frac{\partial B_{z}}{\partial t}+B_{z} \frac{\partial v_{i x}}{\partial x}+v_{i x} \frac{\partial B_{z}}{\partial x}=0
$$

This set of equations can be solved exactly following the procedure suggested by Stenflo et al. [9] for the purely compressive perturbations. We may take

$$
B_{z}=\left(b_{1}+\frac{b_{2} v_{i x}}{2}\right)^{2}
$$

where $b_{1}, b_{2}$ are some arbitrary constants. Using this in Eq. (28), it turns out that Eq. (28) becomes identical to Eq. (27) provided that

$$
c_{1} \equiv \frac{1}{b_{2}^{2}}
$$

The resulting equation for $v_{i x}$ reads

$$
\frac{\partial v_{i x}}{\partial t}+\frac{3}{2} v_{i x} \frac{v_{i x}}{\partial x}+\frac{b_{1}}{b_{2}} \frac{v_{i x}}{\partial x}=0
$$

This equation is identical to Eq. (4) from Ref. [9] obtained for compressive perturbations. It describes steepening of the wave profile and shock wave formation in cold plasma. Having the solution for the speed $v_{i x}$, we can further easily find the magnetic field profile by calculating its components with the help of Eqs. (25, 26, 29). The solution is therefore physically much different compared to Ref. [9] because it implies both compressional and shear components. A similar procedure can be done for soliton description within the reductive perturbation technique used in many references, see the latest in Refs. [26, 27]. 


\section{Conclusion}

It may be concluded that the purely compressive magneto-acoustic perturbations studied in Refs. [1]-[27] and in many others are indeed mathematically possible, yet they are physically very specific and obtained after assuming the trivial value for the perpendicular perturbation. The later is in fact described by a completely identical nonlinear equation derived for the first time in the present work. In general case the compressive and perpendicular perturbations are coupled, the corresponding nonlinear equations contain no small parameters that would support the fact that the perpendicular part is always explicitly omitted. Therefore, in real situations the purely compressive solutions may be far less abundant than expected. After half a century of investigations of the purely compressive mode, a more complete theory is needed which would include both perturbed components of the magnetic field. Such a generalized theory is described here. It contains a rather straightforward derivation of a complete

set of equations, which includes an additional equation for the shear component of the magnetic field. Surprisingly, this equation turns out to be exactly the same as the usual equation for the compressive part of the magnetic field, and it is obtained using the same approximations. This implies that the previously used standard procedures of solving nonlinear equations for the compressive nonlinear magnetic structures can, at least in some cases, be used also in the presented generalized set of equations. One example of that kind is described in the present work.

\section{References}

[1] Adlam J H and Allen J E 1958 Philos. Mag. 3448

[2] Woods L C 1969 Plasma. Phys. 1125

[3] Zaitsev V V 1969 Radiophys. Quant. Electronics 12622

[4] Ohsawa Y and Sakai J I 1988 Solar Phys. 116157

[5] Ohsawa Y and Sakai J I 1988 Solar Phys. 332439

[6] Chiueh T 1989 Phys. Rev. Lett. 63113

[7] Toda M, Ohsawa Y Chiueh T and Jyounouchi T 1995 Phys. Plasmas 23329

[8] Toda M and Ohsawa Y 1997 Solar Phys. 171161

[9] Stenflo L, Shvartsburg A B and Weiland J 1997 Phys. Lett. A 225113

[10] Dogen D, Toida M and Ohsawa Y 1998 Phys. Plasmas 51298

[11] Rau B and Tajima T 1998 Phys. Plasmas 53575

[12] Ödblom A 1998 Phys. Lett. A 24993

[13] Hasegawa H, Irie S, Usami S and Ohsawa Y 2002 Phys. Plasmas 92549

[14] Shukla P K, Eliasson B, Marklund M and Bingham R 2004 Phys. Plasmas 112311

[15] Chaudhuri M, Chakrabarti N and Pal R 2005 Phys. Plasmas 12112101

[16] Sato M and Ohsawa Y 2006 Phys. Plasmas 13063110

[17] Yamauchi K and Ohsawa Y 2007 Phys. Plasmas 14053110

[18] Masood W, Jehan N and Mirza A M 2010 Phys. Plasmas 17132314

[19] Masood W, Rizvi H, Jehan N and Siddiq M 2011 Astrophys. Space Aci. 235405

[20] Shukla P K, Eliasson B and Stenflo L 2011 Phys. Lett. A 3752371

[21] Shukla P K, Eliasson B and Stenflo L 2011 EPL 9545001

[22] Shukla P K, Eliasson B and Stenflo L 2011 Phys. Plasmas 18064511

[23] Chakrabarti C, Maity C and Schamel H 2011 Phys. Rev. Lett. 106145003 
[24] Hussain S and Mahmood S 2011 Phys. Plasmas 18052508

[25] Hussain S and Mahmood S 2011 Phys. Plasmas 18082109

[26] Hussain S, Mushtaq A and Mahmood S 2013 Phys. Scripta 87025502

[27] Shisen R, Shan W, Majid and Cheng Z 2013 Phys. Scripta 87045503 